\title{
Topological pruning enables ultra-low Rayleigh scattering in pressure-quenched silica glass
}

\author{
Yongjian Yang $\mathbb{D}^{1}$, Osamu Homma ${ }^{2}$, Shingo Urata $\mathbb{D}^{2}$, Madoka Ono $\mathbb{i}^{3,4 凶}$ and John C. Mauro $\mathbb{i D}^{1 凶}$
}

Silica glass is the most indispensable material in optical communication applications due to its superior optical properties. The transmission loss of silica glass has been reduced over the past 30 years by continuous efforts toward decreasing density fluctuations by lowering of fictive temperature, e.g., through improvements in processing or doping. A recent study has shown that shrinkage of structural voids by hot compression is a promising way to further decrease the loss. However, an atomic understanding of the pressure effect is still lacking. Here, using molecular simulations, we connect the void shrinkage to topological pruning of silica network. Two physical models predict that the Rayleigh scattering loss of pressure-quenched silica glass can be reduced by $>50 \%$ when the glass is quenched at an appropriate pressure ( $4 \mathrm{GPa}$ in our simulation). Our studies are consistent with available experimental results and demonstrate topologically optimized structure can give desirable properties for optical applications of silica as well as other glasses with similar network structure.

npj Computational Materials (2020)6:139; https://doi.org/10.1038/s41524-020-00408-1

\section{INTRODUCTION}

Silica $\left(\mathrm{SiO}_{2}\right)$ glass is the single most important material for fiber optic communications due to a combination of several favorable properties $^{1}$ including high optical transparency, high tensile/ bending strength, good processability, ability to tune refractive index by doping, and low cost. Because of this unique combination of properties, silica glass has revolutionized global telecommunications through the development of high-bandwidth optical communication networks. During the 1970s, research was vigorously conducted to reduce the loss in silica glass by a factor of a hundred ${ }^{2-4}$. A $10-20 \%$ further decrease in optical attenuation has been achieved, e.g., in fluorine-doped silica glass fiber ${ }^{4,5}$. Interests in low optical loss are also seen in $\mathrm{SiO}_{2}$-based waveguides that are used in planar lightwave circuits ${ }^{6-8}$. Notwithstanding the extreme importance of $\mathrm{SiO}_{2}$ glass in these applications, there is a lack of atomistic level understanding of optical attenuation in the glass. In addition, the lack of recent progress in reducing optical loss is due to limitations in the processing methods that have been considered. More than $80 \%$ of the optical loss in silica glass fiber is due to Rayleigh scattering ${ }^{3,9,10}$, which can be suppressed by reducing the density fluctuations in glass, e.g., by reducing its fictive temperature. The fictive temperature of silica can be reduced by: (a) reducing the cooling rate $^{1} ;$ (b) introducing an annealing process ${ }^{11}$; or (c) reducing the viscosity of the glass by doping with an alkali metal oxide $^{12,13}$. However, reductions in cooling rate or annealing cause a significant decrease in production yield, and compositional variations can lead to an increase in loss due to chemical concentration fluctuations ${ }^{14}$. Recent experimental reports have shown that controlling the pressure history of silica can affect the density fluctuations or heterogeneity and the Rayleigh/X-ray scattering ${ }^{15,16}$. This method of controlling fictive pressure has provide a new train of thought to achieve even lower propagation loss of silica glass. Although the silica glass structural change due to hot compression has been shown to be very different from cold-compressed silica glass with comparable density as well as the pristine silica glass ${ }^{17}$, a systematic understanding of the pressure effect on the silica network structure in terms of homogeneity of the glass at the atomistic level is not available which is required for the design of ideal glass structure with optimal optical properties.

Hence, in this study, we use molecular dynamics (MD) simulations to examine how density fluctuation of $\mathrm{SiO}_{2}$ glass can be suppressed under conditions of varying pressure, including conditions beyond those reported in the experiments ${ }^{15}$. The MD simulations provide a direct probe to the $\mathrm{SiO}_{2}$ network structure which can be compared with experimental results for density and void size within the experimentally achievable applied pressures. The model shows that the optical loss can be improved significantly without using an extremely high pressure. We specifically show that pressure quenching leads to an enhanced homogeneity of the silica glass characterized by a decreasing void size which can be understood using topological pruning picture, bringing a significant reduction in Rayleigh scattering intensity by $\sim 56 \%$ at a quench pressure of $4 \mathrm{GPa}$ in MD. We examine details of the change in homogeneity of $\mathrm{SiO}_{2}$ glass due to hot compression between 0 and $12 \mathrm{GPa}$ and find that it is controlled by two different mechanisms depending on the magnitude of the pressure. While the homogeneity at large pressure is compromised due to an increase of network connectivity, at lower pressures, the dominant homogenization mechanism is related to the topological pruning of the network, which is found to be an effective way for tailoring of $\mathrm{SiO}_{2}$ properties without introducing unwanted defects. These findings provide an atomistic understanding of the pressure-induced network homogenization and also shed light on topological optimization of the silica glass network for optical applications.

\footnotetext{
${ }^{1}$ Department of Materials Science and Engineering, The Pennsylvania State University, University Park, PA 16802, USA. ${ }^{2}$ Innovative Technology Research Center, AGC Inc., 1150 Hazawa-cho, Kanagawa-ku Yokohama, Kanagawa 221-8755, Japan. ${ }^{3}$ Materials Integration Laboratories, AGC Inc., 1150 Hazawa-cho, Kanagawa-ku Yokohama, Kanagawa 221-

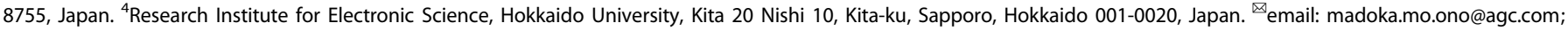
jcm426@psu.edu
} 


\section{RESULTS}

Structure of hot-compressed silica glass from MD

To demonstrate the effect of quench pressure $\left(P_{\mathrm{Q}}\right)$ on silica glass, we have conducted pressure-quench simulations of silica using the recently developed SHIK potential ${ }^{18}$ (see "Methods" for justification of this potential and Supplementary Fig. 1a). Density obtained by experiment is well reproduced by MD simulations using SHIK potential as shown in Fig. 1 (also see Supplementary Fig. 1b). The increase of silica glass density with quench pressure is

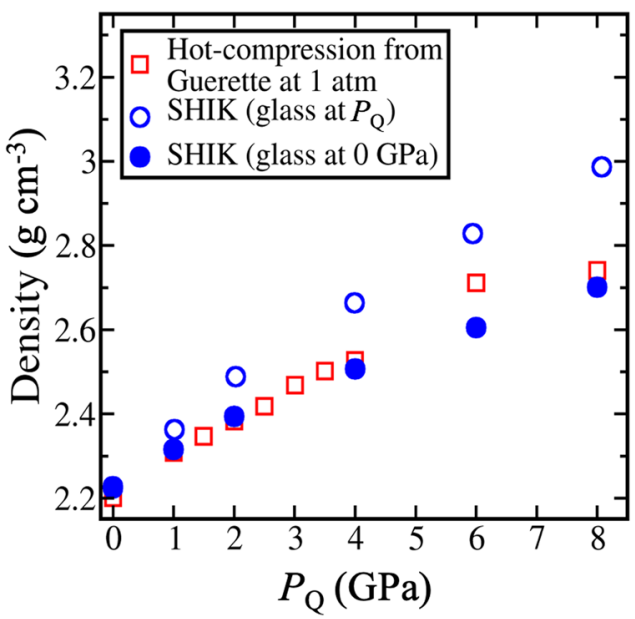

Fig. 1 Densities of the hot-compressed silica glass. The experimental data (red squares) is from Guerette et al. ${ }^{17}$. The compression is done at $1373 \mathrm{~K}$ at each corresponding pressure. The density of the MD simulated glass is on par with the pressure-quenched $\mathrm{SiO}_{2}$ glass in experiments (the open blue circles and the filled blue circles correspond to the simulated glasses before and after the $P_{\mathrm{Q}}$ is released, respectively). For MD simulation, quenching is done from $4000 \mathrm{~K}$, and same as the experiment, the quench pressure $P_{\mathrm{Q}}$ is released down to $1 \mathrm{~atm}$ at room temperature. The standard deviation of the density of MD glasses is around $0.007 \mathrm{~g} \mathrm{~cm}^{-3}$. accompanied by the reduction of structural void size in the glass as illustrated in Fig. 2a. Here, isosurfaces with a minimum distance of $0.1 \mathrm{~nm}$ (about the size of the largest voids in $a$-quartz) from the surface of an atom are visualized as "voids". At $P_{\mathrm{Q}}=0 \mathrm{GPa}$, many of the voids are interconnected in 3D space and their sizes are relatively large. From $P_{\mathrm{Q}}=0$ to $1.0 \mathrm{GPa}$, these voids become more disconnected and their sizes become significantly smaller. At $P_{\mathrm{Q}}=$ 4.0 GPa, only disconnected voids remain, and the number of the voids (with a radius $>0.1 \mathrm{~nm}$ ) are few. At $P_{\mathrm{O}}=8.0 \mathrm{GPa}$, the voids even disappear. Such change persists during the whole cooling procedure (see Supplementary Fig. 2). The void size distributions are shown in Fig. 2b. It shows a rapid extinction of large sized voids (radius $>0.2 \mathrm{~nm}$ ) from ambient pressure up to $2 \mathrm{GPa}$. The largest void size in the silica glass decreases significantly with increasing $P_{\mathrm{Q}}$ as plotted in Fig. 2c. Despite the fact that the experimental data (red points) from positron annihilation lifetime spectroscopy ${ }^{15}$ are only available up to $P_{\mathrm{Q}}=0.2 \mathrm{GPa}$, the largest void size results from $M D$ up to this range show a very good agreement in both the absolute value and the trend.

The significant modification of void morphology and distribution in the pressurized silica glass suggests that some network structure change in the silica glass is triggered by the pressure quench. Here, to characterize the structural change in the $\mathrm{SiO}_{2}$ framework, we make use of the cluster model ${ }^{19,20}$ in which we ignore all $\mathrm{O}$ atoms and consider each $\mathrm{Si}$ atom and its $\mathrm{Si}$ coordination sequence $\mathrm{CS}=\left\{N_{\mathrm{k}}\right\}=N_{1}, N_{2}, \ldots, N_{k}$, where $N_{k}$ indicates the number of $\mathrm{Si}$ atoms in "sphere" $k$ which are connected to Si atoms in sphere $k-1$. A cutoff of $3.4 \AA$ (see the "Methods") is used to find the neighbor Si atoms of each $\mathrm{Si}$ atom. Note that here the $N_{1}$, i.e., $\mathrm{CN}_{\mathrm{Si}-\mathrm{Si}}$ is not the same as the $\mathrm{Si}$ coordination, $\mathrm{CN}_{\mathrm{Si}-\mathrm{O}}$ (i.e., the coordination number of $\mathrm{Si}-\mathrm{O}$ ), often referred in experiments ${ }^{17,21,22}$, which is very close to 4 in our simulations $\left(\mathrm{CN}_{\mathrm{Si}-\mathrm{O}} \leq 4.05\right.$ at $P_{\mathrm{Q}} \leq 8 \mathrm{GPa}$, see Supplementary Fig. 4) and consistent with other simulations ${ }^{23}$. It has also been shown ${ }^{23,24}$ that a slower cooling rate such as the $1 \mathrm{~K} \mathrm{ps}^{-1}$ used in current study tends to generate a $\mathrm{CN}_{\mathrm{Si}-\mathrm{O}}=4$ network when the $\mathrm{SiO}_{2}$ glass density is less than $2.7 \mathrm{~g} \mathrm{~cm}^{-3}$ (or $P_{\mathrm{Q}}<8 \mathrm{GPa}$ ). An example of $\mathrm{CS}$ is shown in the inset of Fig. $3 \mathrm{~b}$ (left panel) wherein

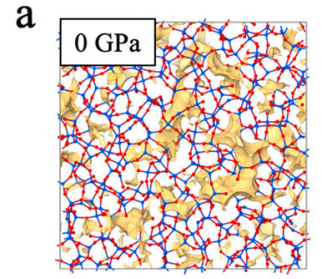

b

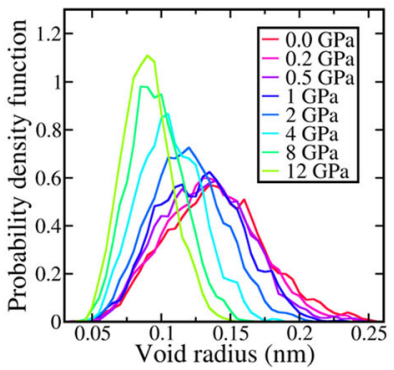

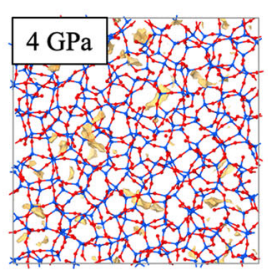

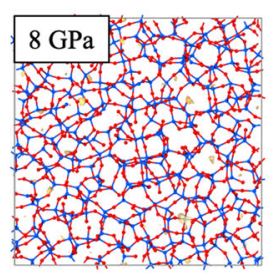

C

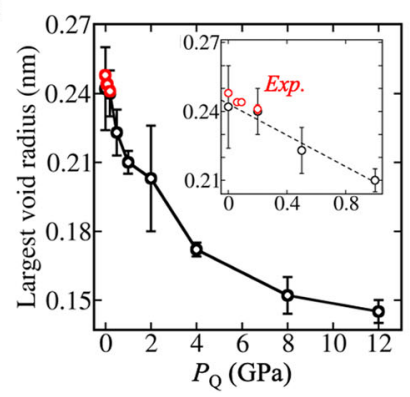

Fig. 2 Pressure effects on voids in silica glass. a Morphologies of voids in four silica glass samples pressure-quenched at $0,1,4$, and $8 \mathrm{GPa}\left(P_{\mathrm{Q}}\right.$ has been released). The yellow regions are isosurfaces with a minimum distance of $0.1 \mathrm{~nm}$ from the surface of any atoms in the glass. In other words, voids with a radius $<0.1 \mathrm{~nm}$ are not shown. The blue and red atoms are Si and O, respectively. Only a slice of the glass $(\sim 10 \AA$ thick) is visualized for clearness. b Void radius distributions at different pressures. The curves are smoothed using a moving average with a step of $0.05 \mathrm{~nm}$. c Variation of the largest void radius from both experiments (red, up to $0.2 \mathrm{GPa}$ ) and MD simulations. The inset is a close-up view of the pressure region from 0 to $1 \mathrm{GPa}$ where the dashed line is a guide for eyes to show the consistent trends between the simulation results and the positron annihilation lifetime spectroscopy experiments ${ }^{15}$. The error bar is calculated from the standard deviation of multiple samples. 
a
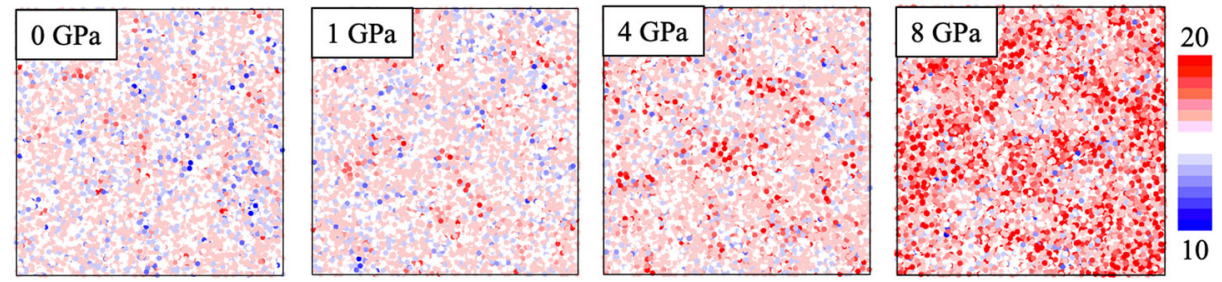

b
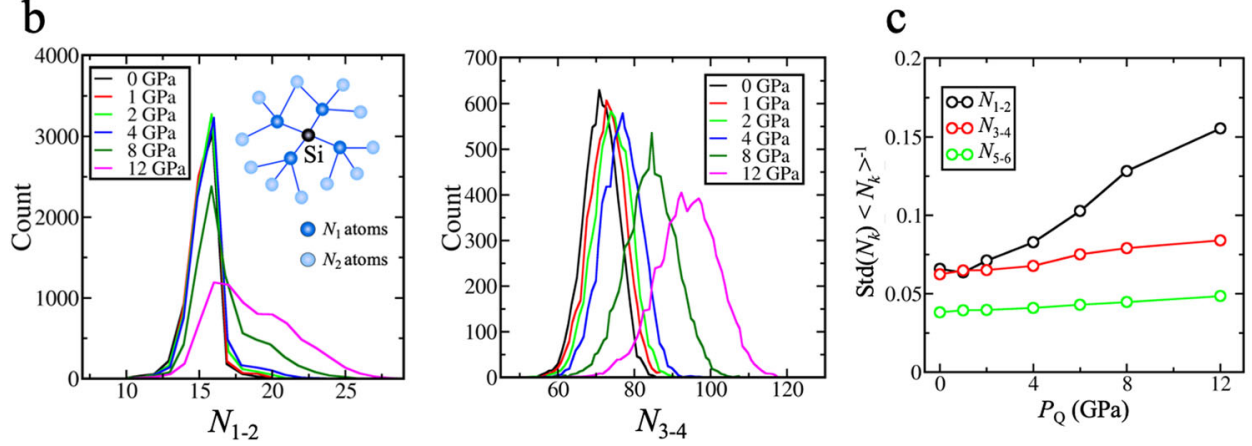

Fig. 3 Local Si neighbor number in silica glass. a Spatial distribution of $N_{1-2}\left(=N_{1}+N_{2}\right)$ of each Si atom in the silica glass samples pressure quenched at $P_{\mathrm{Q}}=0,1,4$, and $8 \mathrm{GPa}$. The color scheme is shown on the right hand. b Statistical distribution of $N_{1-2}$ and $N_{3-4}$ in the pressurequenched silica glass. The inset in $\mathbf{b}$ gives an example in which the central $\mathrm{Si}$ atom (black) has four first neighbor $\mathrm{Si}$ atoms (blue, $\left.N_{1}=4\right)$ and eleven second neighbor $\mathrm{Si}$ atoms (light blue $N_{2}=11$ ). c Variation of normalized standard deviation (Std) of $N_{k}$ as a function of the quench pressure.

the central Si atom has $N_{1}=4$ and $N_{2}=11$. The spatial distribution of $N_{1-2}$ (i.e., $N_{1}+N_{2}$ ) in the silica glass and its statistics are shown in Fig. $3 a, b$ from which a significant increase in the dispersion of $N_{1-2}$ appears when $P_{\mathrm{Q}}$ increases from 4 to $8 \mathrm{GPa}$. Likewise, the dispersion of the $\mathrm{N}_{3-4}$ (Fig. $3 \mathrm{~b}$, right panel) is also seen to increase more significantly at $P_{\mathrm{Q}}>4 \mathrm{GPa}$. The normalized standard deviation (Std $\left(N_{k}\right)<N_{k}>^{-1}$ ) in $N_{k}$ is shown in Fig. 3c, where a larger increase of variation in the $\mathrm{Si}$ coordination sequence is seen in $N_{1-2}$ (the 1st and 2nd neighboring $\mathrm{Si}$ atoms) than in $N_{3-4}$ and so forth in $N_{5-6}$. The change in the 1 st (locating at $\sim 3 \AA$ away) and the 2nd (locating at $\sim 5 \AA$ away) neighboring $\mathrm{Si}$ atoms is consistent with the major variation in void size as shown in Fig. 2 particularly in those voids whose diameter is between 2 and $4 \AA$ (note the atomic radius of $\mathrm{Si}(0.26 \AA)$ and $\mathrm{O}(1.35 \AA)$ are taken into account). The large variation in $N_{k}$ in Fig. $3 \mathrm{~b}$, especially in $N_{1-2}$ also suggests that the homogeneity of the silica glass would deteriorate when $P_{\mathrm{Q}}$ is too high and different $\mathrm{CN}_{\mathrm{Si}-\mathrm{Si}}$ appears.

\section{Calculation of Rayleigh scattering of densified silica glass}

As a result of decreasing void size, Rayleigh scattering is expected to be significantly reduced with improvement of the glass homogeneity. The Rayleigh scattering can be quantified via either Mie theory or density fluctuations (see "Methods"). The Rayleigh scattering intensity $R_{\mathrm{sp}}$ of all voids can be calculated using the following equation based on the Mie theory ${ }^{25}$ which sums contribution from all $N$ voids in the material by treating them as scattering spherical particles,

$R_{\mathrm{sp}}=\frac{1}{V} \sum_{i=1}^{N} \frac{2}{3} \pi^{5}\left(\frac{n^{2}-1}{n^{2}+2}\right)^{2} \frac{D_{i}^{6}}{\lambda^{4}}$,

where $V$ is the volume of the material, $n$ is the relative refractive index, $D_{i}$ is the diameter of the $i$ th void and $\lambda$ is the wavelength the incoming light. $R_{\text {sp }}$ calculated from all voids (see "Methods" and Fig. $2 \mathrm{~b}$ ) is plotted against $P_{\mathrm{O}}$ in Fig. $4 \mathrm{a}$ by the blue curve. The plot shows a clear suppression of $R_{\mathrm{sp}}$ (e.g., $\sim 57 \%$ from 0 to $4 \mathrm{GPa}$ or $\sim 65 \%$ when $8 \mathrm{GPa} \leq P_{\mathrm{Q}} \leq 12 \mathrm{GPa}$ ). While it is difficult to directly obtain the Rayleigh scattering coefficient $a_{\mathrm{scat}}$ from $R_{\mathrm{sp}}$, we have attempted to convert $R_{\mathrm{sp}}$ to $a_{\text {scat }}$ at $1.55 \mu \mathrm{m}$ via two $\mathrm{SiO}_{2}$ samples from MD whose largest pore sizes are similar as two $\mathrm{SiO}_{2}$ samples from experiments ${ }^{15}$ whose $a_{\text {scat }}$ values are known (see "Methods"). Such obtained $a_{\text {scat }}$ value for each $\mathrm{MD} \mathrm{SiO}_{2}$ sample is shown using the right axis of Fig. 4a. The inset of Fig. 4a compares the MD results with the available experimental $a_{\text {scat }}$ available up to $P_{\mathrm{Q}}=$ $0.2 \mathrm{GPa}^{15}$ where a suppression in $a_{\text {scat }}$ is seen in both cases. The amount of change in $a_{\text {scat }}$ from MD seems to be smaller than the experiments at $0.2 \mathrm{GPa}$, which may result from several factors: (1) the inaccuracy of the conversion from $R_{\mathrm{sp}}$ to $a_{\text {scat }}$ (only the largest pore is considered), (2) the simple treatment of pore as spherical particles in the Mie theory while their shapes are irregular as shown in Fig. 2a, and/or (3) the much shorter time scale used in MD under high pressure than experiments.

Alternatively, we may calculate $a_{\text {scat }}$ from the density fluctuations of the silica glass. As noted in "Methods", $a_{\text {scat }}$ can be written in terms of density fluctuations $\frac{\left\langle N^{2}\right\rangle-\left\langle N^{2}\right\rangle}{\langle N\rangle}$ as $^{10,26}$ :

$a_{\text {scat }}=\left(10 \log _{10}\right.$ e) $\frac{8 \pi^{3}}{3 \lambda^{4}} n^{8} p^{2} \frac{\left\langle N^{2}\right\rangle-\left\langle N^{2}\right\rangle}{\langle N\rangle \bar{n}}$,

where $\lambda$ is the wavelength, $n$ is refractive index, $p$ is the photoelastic coefficient, and $\bar{n}$ is the number density of particles. $N$ is the number of particles in volume $V$ and the angle brackets indicate an average. The density fluctuation $\frac{\left\langle N^{2}\right\rangle-\langle N\rangle^{2}}{\left\langle N^{2}\right\rangle}$ can be calculated from density/volume variation of multiple samples melt-quenched using, e.g., the NPT ensemble. Given the empirical relation between $n$ and $\rho$ (the density of a glass in $\left.\mathrm{g} \mathrm{cm}^{-3}\right)^{17,27}$, i.e., $n=(\rho-2.21) \times 0.193+1.46$, the photoelastic constant $p$ for an isotropic solid can be calculated as $\frac{2 \rho}{n^{3}} \frac{\partial n}{\partial \rho} 10,28$ which, when substituted in Eq. 2, would reduce the dependence on $n$ from the eighth power to the second power. Use of this empirical relation is justified because the relative error of $n$ is $<2 \%$ based on ref. ${ }^{27}$ which would at most cause a relative error of $\sim 4 \%$ in $a_{\text {scat }}$. $a_{\text {scat }}$ as a function of quench pressure using Eq. (2) is shown in Fig. 4b. The Rayleigh scattering is found to be suppressed at $P_{\mathrm{Q}}=$ $4 \mathrm{GPa}$ with a reduction of $56 \%$ compared with at $0 \mathrm{GPa}$ which is very close to the reduction in $R_{\mathrm{sp}}(57 \%)$ from Fig. $4 \mathrm{a}$. Specifically, a scattering loss $\sim 0.081 \mathrm{~dB} \mathrm{~km}^{-1}$ due to Rayleigh scattering can be achieved at $4 \mathrm{GPa}$, which is in the same ballpark as the Rayleigh 

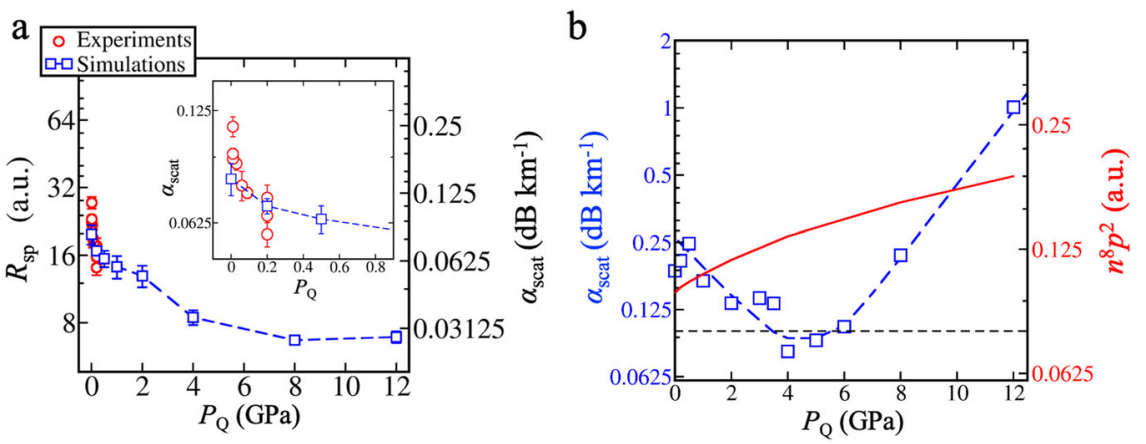

Fig. 4 Rayleigh scattering of pressure-quenched $\mathrm{SiO}_{2}$ glasses at a wavelength of $\mathbf{1 . 5 5} \boldsymbol{\mu m}$. a Calculated $R_{\mathrm{sp}}$ (left axis) at different $P_{\mathrm{Q}}$ using the Mie theory and the corresponding $a_{\text {scat }}$ at $\lambda=1.55 \mu \mathrm{m}$ (right axis). Experimental results from ref. ${ }^{15}$ are also included for comparison. Note that the experimental data in Fig. 3a contain $\mathrm{SiO}_{2}$ glasses quenched using the same pressure but different holding times or different glass transition temperature; therefore, multiple values exist at $P_{\mathrm{Q}}=0.0$ and $0.2 \mathrm{GPa}$. The error bars are estimated by the difference between the largest $R_{\mathrm{sp}}$ and the smallest $R_{\mathrm{sp}}$ at each $P_{\mathrm{Q}}$. $\mathrm{b}$ Calculated $a_{\mathrm{scat}}$ and $n^{8} p^{2}$ at different quench pressures using density fluctuations. Each $a_{\mathrm{scat}}$ is calculated from density variation of eighteen samples (see "Methods"). Additional data from $\mathrm{SiO}_{2}$ samples with different $P_{\mathrm{O}}$ are added to obtain the trend for $a_{\text {scat }}$. The blue dashed line is an eye guide to show the trend of $a_{\text {scat }}$. The black dashed line corresponds to $0.1 \mathrm{~dB} \mathrm{~km}^{-1}$.

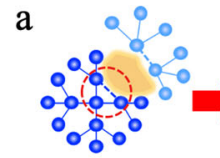

(i)

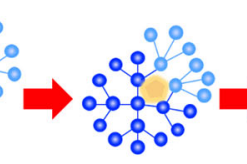

(ii)

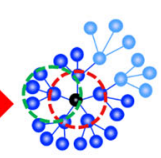

(iii)

$\mathrm{b}$

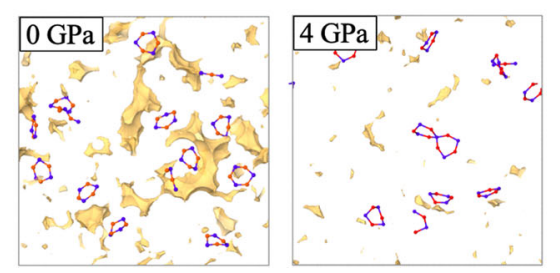

c

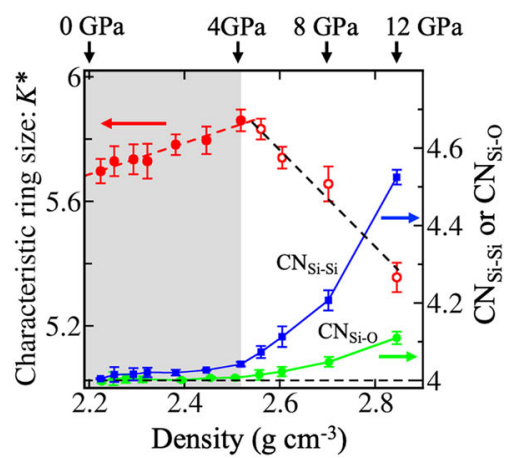

Fig. 5 Homogenization mechanism of silica glass. a Configurational change to account for the homogenization effect (via elimination of a

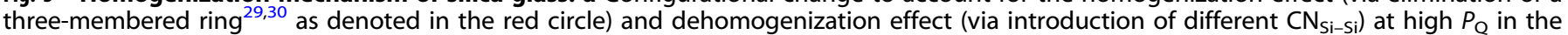
$\mathrm{SiO}_{2}$ glass. The yellow area denotes a void whose size decreases after the exemplary three-membered ring is eliminated. All spheres denote the Si atoms (the $\mathrm{O}$ atoms are not considered). b Proximity of free space to 3-memberd rings (Si: blue atoms and O: red atoms) in silica glasses whose $P_{\mathrm{Q}}=0$ and $4 \mathrm{GPa}$. The yellow regions are isosurfaces with a minimum distance of $0.11 \mathrm{~nm}$ from the surface of any atoms in the glass. c Characteristic ring size $K^{*}$ (red) and $\mathrm{CN}_{\mathrm{Si}-\mathrm{Si}}$ or $\mathrm{CN}_{\mathrm{Si}-\mathrm{O}}$ at different densities. $\mathrm{CN}_{\mathrm{Si}-\mathrm{Si}}$ is defined as the average number of first Si neighbor atoms to a central one using a cutoff of $3.4 \AA$. The dashed red line and black dashed line are guides for eyes. Multiple samples were used to calculate the standard deviation for the error bar.

scattering loss of conventional/hot-compressed silica in experiments ${ }^{11,15}$ and is extremely low given the much higher cooling rate used in $\mathrm{MD}\left(1 \mathrm{~K} \mathrm{ps}^{-1}\right)$. However, a further increase of $P_{\mathrm{Q}}$ to $12 \mathrm{GPa}$ will instead enhance the scattering by more than 10 times, in contrast with Fig. 4a, suggesting that factors other than voids should be considered at higher pressure. Figure $4 \mathrm{~b}$ also shows the change of the $n^{8} p^{2}$ term in Eq. (2) with a monotonic increasing trend with $P_{\mathrm{Q}}$ (e.g., an increase of $37 \%$ is seen from 0 to $4 \mathrm{GPa}$ ), confirming that the lowering of $a_{\text {scat }}$ comes solely from change of glass homogeneity. We note here that even though it seems that predictions using Mie theory gives smaller $a_{\text {scat }}$ than those from density fluctuation, both Fig. $4 a$, b show that a reduction of Rayleigh scattering by $\sim 56 \%$ can be achieved using a moderate quench pressure $\sim 4 \mathrm{GPa}$. The calculated value of $a_{\text {scat }}$ using voidscattering and density fluctuation models are, respectively, 0.069 and $0.21 \mathrm{~dB} \mathrm{~km}^{-1}$ under $0.2 \mathrm{GPa}$. Although the former value matches well with the experimental results $\left(\sim 0.06-0.07 \mathrm{~dB} \mathrm{~km}^{-1}\right)$ as shown by the inset of Fig. 4 a, we believe the latter value is more accurate when considering the high cooling rate and the short $P_{\mathrm{Q}}$ holding time used in the MD simulations. The fictive temperatures $(>2100 \mathrm{~K})$ of the $\mathrm{SiO}_{2}$ glasses in $\mathrm{MD}$ simulations are several hundred degrees higher than the experimental samples, suggesting that an extremely low Rayleigh scattering coefficient may be achieved by tailoring the thermal history of the glass in combination with its pressure history as reported by ref. ${ }^{15}$. The results of $a_{\text {scat }}$ calculation using void-scattering and density fluctuation both clearly indicate that, by increasing $P_{\mathrm{Q}}$ from 0.0 to $4 \mathrm{GPa}$, a continuous decrease of $a_{\text {scat }}$ is expected. Such improvement in $a_{\text {scat }}$ should come from amelioration of homogeneity of silica glass as evidenced in Fig. 2 .

Understanding the homogenization of silica glass

To understand the homogenization mechanism of the silica network as a result of $P_{\mathrm{Q}}$, we examine the structural differences among those glasses. We evaluate the change of ring size distribution (whose calculation method is described in "Methods" and see Supplementary Fig. 3) for the simulated silica glass. For $P_{\mathrm{Q}}$ $<6 \mathrm{GPa}, \mathrm{CN}_{\mathrm{Si}-\mathrm{O}}$ in the silica glass remains close to 4.0 and the $\mathrm{Si}-\mathrm{Si}$ distance scarcely changes (see Fig. 5c, Supplementary Fig. 4 and Supplementary Methods). The improvement in $\mathrm{SiO}_{2}$ homogeneity can be understood using the topological pruning mechanism

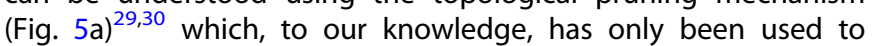
account for the densification of $\mathrm{SiO}_{2}$ network. According to refs. ${ }^{29,30}$, ring formation reduces the density of the network by pruning a calculable number of tetrahedrally coordinated atoms 
a

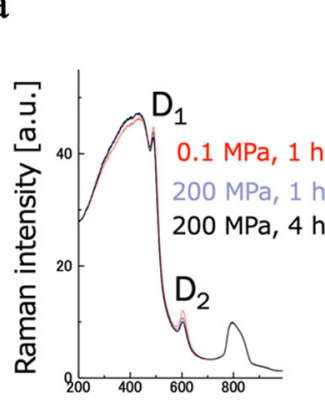

Wavenumber $\left[\mathrm{cm}^{-1}\right]$

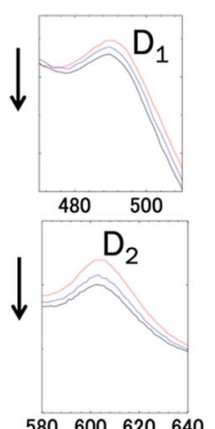

Wavenumber $\left[\mathrm{cm}^{-1}\right]$ b

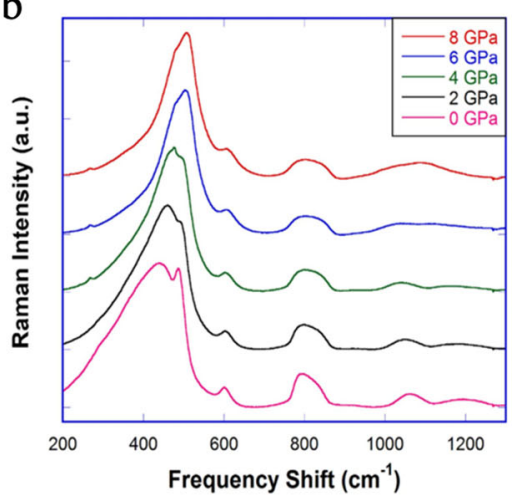

Fig. 6 Raman spectrum of silica glass. a Raman spectrum of three pressure-quenched silica glass samples prepared at $0.1 \mathrm{MPa}$ for $1 \mathrm{~h}, 0.2 \mathrm{GPa}$ for $1 \mathrm{~h}$, and $0.2 \mathrm{GPa}$ for $4 \mathrm{~h}$, all at $2073 \mathrm{~K}$. The variations of the relative intensity of the $D_{1}$ and $D_{2}$ peaks as a function of time are enlarged for clarity on the right. $\mathbf{b}$ Raman spectrum of pressure-quenched $\mathrm{SiO}_{2}$ glasses from higher pressures at $1373 \mathrm{~K}$ by Guerette et al. ${ }^{17}$.

(Si atoms) and the relative ability of different sized rings to reduce the network density decreases exponentially with the ring size $K$. Therefore, a higher density of $\mathrm{SiO}_{2}$ can be achieved by eliminating smaller rings as illustrated in Fig. 5a: (i)-(ii) and increasing the characteristic ring size $K^{*}$ (see Supplementary Methods for calculation of $\left.K^{*}\right)$. At $P_{\mathrm{Q}} \leq 4 \mathrm{GPa}$, small ring ratios $(K \leq 7)$ are found mostly to decrease with $P_{\mathrm{Q}}$ (see Supplementary Fig. 3B, D) and $K^{*}$ approaches 6 as shown in Fig. 5a. Note a linear increase of $K^{*}$ in the simulated glass with pressure at $P_{\mathrm{Q}} \leq 4 \mathrm{GPa}$ is observed, which means that the glass network should become more homogeneous in the sense that its $K^{*}$ is closer to that of the quartz (whose $K^{*}$ is between 6 and 8) whose $a_{\text {scat }}$ is extremely small $\left(<0.01 \mathrm{~dB} \mathrm{~km}^{-1}\right.$ using $k_{\mathrm{B}} T \beta_{\mathrm{T}}{ }^{31}$ where $T=300 \mathrm{~K}$ and $\left.\beta_{\mathrm{T}}=2.68 \times 10^{-11} \mathrm{~Pa}^{32}\right)$. In order to further correlate such change to the void result (Fig. 2), we propose an alternative way to account for the homogeneity improvement via the proximity of free space, i.e., voids, to smaller rings $(K \leq 7)$ which, partially, can be pruned to densify the glass. An example is given in Fig. 5a: (i)-(ii) in which the free space (yellow region) near the 3-member ring shrinks after the ring is pruned to allow additional Si atoms (light blue) to connect with the group of dark blue Si atom. Similar mechanism can also occur at the proximity of 4 and 5 membered rings. The proximity of the free space to 3-membered rings is evidenced in Fig. $5 b$ (also for $K=4$ in Supplementary Fig. 5) at $0 \mathrm{GPa}$. Unlike the shrinkage of pores due to distortion of the rings under pressure which tends to recover once $P_{\mathrm{O}}$ is released, this achievement in homogenization requires breaking and forming of bonds which shall persist after $P_{\mathrm{Q}}$ is released at the room temperature.

On the other hand, large rings $(K>7)$ increase, and particularly, small rings $(K<6)$ are found to significantly rebound at $P_{\mathrm{Q}}>4 \mathrm{GPa}$ (see Supplementary Fig. $3 \mathrm{~b}$ ). This change in the constituent ratio of the ring size comes from the increase of $\mathrm{CN}_{\mathrm{Si}-\mathrm{Si}}$ in the silica glass as shown in Fig. 5a: (ii)-(iii), leading to an increase in density fluctuation as shown by the different number of Si atoms enclosed by the red and green circles in Fig. 5 a (iii) and the local Si neighbor number shown in Fig. 3. We note that the increase in the constituent ratio of small rings is also seen before $P_{\mathrm{Q}}$ is released (Supplementary Fig. 3c), which is consistent with experimental results under high pressure by Hemley et al. ${ }^{33}$ and ab initio calculations by Trave et al. ${ }^{34}$ and Karki et al. ${ }^{35}$ of the silica liquid.

Although there is no consensus on the change of the populations of the 3- and 4-membered rings with pressure in silica glass/liquid from previous MD simulation results ${ }^{17,29,34-36}$ probably due to the usage of different simulation settings, e.g., the interatomic potential, the cooling rate, the pressure condition, the ring concepts, etc., our ring statistics seem to be supported experimentally by the change of the two defect peaks, $D_{1}$ and $D_{2}$, arising from symmetric stretch of regular 4-membered rings and 3-membered rings ${ }^{37,38}$. Figure $6 a$ shows our Raman measurements from the samples used in ref. ${ }^{15}$. Both $D_{1}$ and $D_{2}$ peaks are found to decrease in intensity without any frequency shift at higher pressure and at longer holding time, when the silica glasses are quenched from $2073 \mathrm{~K}$ with one at ambient pressure and two others held at $200 \mathrm{MPa}$ for 1 and $4 \mathrm{~h}$ prior to the quench, suggesting a smaller population of the two types of the rings due to hot compression. The weakening (between 0 and $4 \mathrm{GPa}$ ) of the two peaks is also observed by the Raman spectrum results of $\mathrm{SiO}_{2}$ glass by Guerette et al. ${ }^{17}$ (Fig. 6b) quenched from a lower temperature $1373 \mathrm{~K}$. Instead, at large pressures, e.g., $8 \mathrm{GPa}$, the intensities of the two peaks seem to be enhanced according to Fig. $6 \mathrm{~b}$ using the right-most point as the reference. By comparison, the intensity of the $D_{2}$ peak of silica glass subjected to cold compression increases monotonically with the maximal pressure reached $^{39}$. Here, we have several remarks. First, although experiments show that the $\mathrm{CN}_{\mathrm{Si}-\mathrm{O}}$ of hot-compressed silica remains at close to 4 with $P_{\mathrm{Q}}$ up to $8 \mathrm{GPa}^{17,21}$, they do not preclude $\mathrm{CN}_{\mathrm{Si}-\mathrm{Si}}$ from being higher than 4 as shown in Fig. $5 \mathrm{C}$. Second, due to the much higher cooling rate used in MD, it is likely that the increase of $\mathrm{CN}_{\mathrm{Si}-\mathrm{O}}$ and $\mathrm{CN}_{\mathrm{Si}-\mathrm{Si}}$ may be postpone at some $P_{\mathrm{Q}}$ value larger than $4 \mathrm{GPa}$ if a slow cooling rate is used. However, heterogeneity shall occur as long as a coexistence of different $\mathrm{CN}_{\mathrm{Si}-\mathrm{O}} / \mathrm{CN}_{\mathrm{Si}-\mathrm{Si}}$ starts to appear which will enhance optical scattering ${ }^{16}$. Lastly, based on the works by Bridgman ${ }^{40-42}$, Vukcevich $^{43}$ has shown a maximal compressibility is reached for cold-compressed silica glass under a pressure between 3 and $4 \mathrm{GPa}$. However, this is different from the observed lowest density fluctuation of the hot-compressed silica glass in our simulations which corresponds to a minimal compressibility at around $4 \mathrm{GPa}$ (see Supplementary Fig. 1d).

\section{DISCUSSION}

The change of density fluctuations in $\mathrm{SiO}_{2}$ glass with $P_{\mathrm{Q}}$ can now be clearly understood in terms of the two topological modification of $\mathrm{SiO}_{2}$ network. At $P_{\mathrm{Q}}<4 \mathrm{GPa}$, decrease of population of smaller rings and increase of larger ring population drive the network to be closer to a perfect Bethe lattice (Fig. 5a) whose density fluctuations is extremely low (all lattice points have equal chemical sequence). Such tendency of $\mathrm{SiO}_{2}$ structure to approach the Bethe lattice can lead to a decrease in void size (Fig. 5a). At higher $P_{\mathrm{Q}}$, the equality of the Bethe lattice points is undermined by

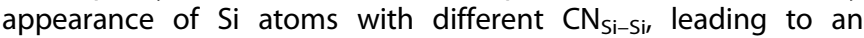
increased density fluctuation. Thus, the highest homogeneity of silica glass is achieved at some intermediate value of $P_{\mathrm{Q}}(\sim 4 \mathrm{GPa})$ in our MD simulations. Lastly, the high cooling rate used in MD may shift the critical pressure to a smaller value above which 
$\mathrm{CN}_{\mathrm{Si}-\mathrm{O}}$ and $\mathrm{CN}_{\mathrm{Si}-\mathrm{Si}}$ start to increase rapidly. This suggests that further improvement in Rayleigh loss can be achieved by using a slower cooling rate at a higher $P_{\mathrm{Q}}$.

Previous MD results by Kaiki et al. ${ }^{35}$ show that the Grüneisen parameter and heat capacity of the liquid $\mathrm{SiO}_{2}$ have distinct values in this low-pressure regime $(P \leq 4 \mathrm{GPa})$. While a complete analysis of these properties is beyond the scope of this study, the $\mathrm{SiO}_{2}$ structure between 0 and $4 \mathrm{GPa}$ changes solely in the ring structure without introduction of defects, e.g., under- or over-coordinated $\mathrm{Si} / \mathrm{O}$ atoms, with adverse effect on optical properties. Our study, therefore, shows a topological way of improving the optical properties of silica glass which may be applicable in other network glasses such as $\mathrm{GeO}_{2}$ and $\mathrm{B}_{2} \mathrm{O}_{3}$. Lastly, while it is tempting to connect the characteristic ring size $K^{*}$ to the void size in $\mathrm{SiO}_{2}$, according to our results (Figs. 2b, 5b), they vary with $P_{\mathrm{Q}}$ in opposite directions at $P_{\mathrm{Q}}<4 \mathrm{GPa}$, which suggests that the ring pruning should play a critical role in the homogenization.

To sum up, we have shown that, using the pressure-quench method and two different methods to calculate Rayleigh loss, the homogeneity of silica glass can be significantly improved, achieving a reduction in Rayleigh scattering by more than $50 \%$. Given the different simulation conditions compared to experiments, the improvement in loss is expected to be even greater if lower cooling rates are used. Such improvement is due to the homogenization in the pressurized silica network. Specifically, at $P_{\mathrm{Q}} \leq 4 \mathrm{GPa}$, the reduction in density fluctuation results from a change in intermediate-range order that is supported by Raman spectrum and ring structure analysis in our MD simulations and can be understood as an approaching of the network's characteristic ring size to a perfect Bethe lattice. Higher values of $P_{\mathrm{Q}}$, however, are found to potentially bring about coexistence of different network coordinations which will undermine the homogeneity of the network. Our findings have shown, through an atomistic understanding, how the homogeneity of $\mathrm{SiO}_{2}$ is improved by pressure; topological pruning from decrease of small rings can drive the network to be more like a Bethe lattice and at the same time decrease those voids close to the pruned rings without introducing unwanted defects. This provides not only the detailed understanding of the structural change induced by hot compression, but also an example of ideal $\mathrm{SiO}_{2}$ glass structure of high homogeneity which is desirable for optical applications. Such ideal glass structure is useful not only for silica glass, but also for other types of inorganic glass with similar network structure such as $\mathrm{GeO}_{2}$. Although a high $P_{\mathrm{Q}}$ at high temperature is still technically challenging, the ideal glass structure might be approachable using other techniques such as high-pressure chemical vapor deposition $^{44,45}$, which might be applied to improve the optical transparency of light waveguide.

\section{METHODS}

\section{Molecular dynamics simulations}

Silica glass samples were generated by randomly distributing 3000 atoms in a cubic box and melted in the liquid state at $4000 \mathrm{~K}$ at different pressures for $100 \mathrm{ps}$, followed by a quenching process to $300 \mathrm{~K}$ at a rate of $1 \mathrm{~K} \mathrm{ps}^{-1}$. It has also been shown ${ }^{23,24}$ that a slower cooling rate such as the $1 \mathrm{~K} \mathrm{ps}^{-1}$ used in current study tends to generate a $\mathrm{CN}_{\mathrm{Si}-\mathrm{O}}=4 \mathrm{SiO}_{2}$ network when the $\mathrm{SiO}_{2}$ glass density is less than $2.7 \mathrm{~g} \mathrm{~cm}^{-3}\left(P_{\mathrm{Q}}<8 \mathrm{GPa}\right.$ according to Fig. 1). The quench pressure $P_{\mathrm{O}}=0-12 \mathrm{GPa}$ is maintained using a Nosé-Hoover thermostat/barostat ${ }^{46-48}$ in the isothermal-isobaric ensemble (NPT). Larger samples of 24,000 atoms were used to get the cooling curves (e.g., Fig. 1) and measure the density fluctuation. After the meltquench, the pressure is reduced to $1 \mathrm{~atm}$ in $100 \mathrm{ps}$ followed by an additional run for $100 \mathrm{ps}$ at $1 \mathrm{~atm}$. The SHIK potential ${ }^{18}$ used for the atomic interaction has been found to accurately reproduce radial distribution function (RDF) of silica liquid ${ }^{18}$, vibrational density of states ${ }^{18}$, and the density of glass at $300 \mathrm{~K}$ at various pressures. The Si speciation in liquid $\mathrm{SiO}_{2}$ predicted by this potential also compares well with ab initio calculation and TS potential considering $\mathrm{O}$ polarizability (see
Supplementary Fig. 1c). For comparison, BKS potential $\left.\right|^{49,50}$ broadly applied for $\mathrm{SiO}_{2}$ simulations is also used to generate silica glass by pressure quench of the liquid from 7000 to $300 \mathrm{~K}$ using the same cooling rate (see Supplementary Fig. 1b). Note a higher cooling rate is used in ref. ${ }^{24}$, making the resulted glass densities different from us. All MD simulations and structure visualizations are carried out using LAMMPS $^{51}$ and OVITO ${ }^{52}$, respectively.

\section{Structural analysis}

A cutoff of $2.2 \AA \AA$ is used for Si-O pair, and $3.4 \AA$ for Si-Si pair for bond angle and coordination analysis. These values are the position of the first minimum in the corresponding RDFs. The void and its distribution was obtained using Zeo $++{ }^{53,54}$. Shannon radii ${ }^{55}$ for Si, $0.26 \AA$ and $\mathrm{O}, 1.35 \AA$ are used to define the surface of an atom and for the void analysis, which are very close to the radii determined in ref. ${ }^{22}$. The void radius distribution from Zeo++ does not give the number of voids. To calculate the total amount of voids, we use the surface area function in Zeo++ to obtain the number of voids whose radii are larger than a critical value so that they comprise of the largest $\sim 20 \%$ voids using Fig. $2 \mathrm{~b}$. The total number of voids is then calculated by dividing this number with this percent. The R.I.N.G.S. code ${ }^{56}$ was used to determine the primitive ring distribution.

\section{Raman spectroscopy}

Raman spectra were collected with a Raman microscope (Nicolet Almega, Thermo Fisher Scientific Inc., Yokohama, Japan) in backscattering geometry. The excitation wavelength was $532 \mathrm{~nm}$. Polished plates with dimensions of $15 \times 15 \times 1 \mathrm{~mm}$ were used for the measurements. $100 \times$ microscope and $1800 \mathrm{Imm}^{-1}$ grating were used. Data were taken with the exposure time set as $60 \mathrm{~s}$, with accumulation of 10 times. All measurements were conducted at room temperature (around $300 \mathrm{~K}$ ).

\section{Calculation of Rayleigh scattering ( $R_{\mathrm{sp}}$ and $\left.a_{\mathrm{scat}}\right)$}

The $R_{\mathrm{sp}}$ can be directly calculated using Eq. (1) and the total number of voids and the void distribution obtained from Zeo $++{ }^{53,54}$. Two methods were used to calculate the Rayleigh attenuation $a_{\text {scat }}$. In method I (see Eq. (3)), the following equation is assumed to derive $a_{\text {scat }}$ from $R_{\text {sp }}$ for the MD simulated silica glasses,

$a_{\text {scat }}=A \log _{10}\left[1-B R_{\mathrm{sp}}\right]=C \cdot R_{\mathrm{sp}}$,

where $A, B$, and $C$ are constants and the second equation is valid when the term inside the logarithmic function is close to 1 . Two $\mathrm{SiO}_{2}$ samples pressure-quenched at 0 and $0.2 \mathrm{GPa}$ in the current $\mathrm{MD}$ simulation whose largest pore sizes $(0.2435$ and $0.2415 \mathrm{~nm})$ and densities $(2.210$ and $2.215 \mathrm{~g}$ $\left.\mathrm{cm}^{-3}\right)$ are similar to two of the experimental samples in ref. ${ }^{15}(0.2436 \mathrm{~nm}$ and $0.2414 \mathrm{~nm}$, and $2.230 \mathrm{~g} \mathrm{~cm}^{-3}$ and $2.256 \mathrm{~g} \mathrm{~cm}^{-3}$ ) whose $a_{\text {scat }}$ are known. $C$ are found to be $4.14 \times 10^{-3} \mathrm{~dB} \mathrm{~km}^{-1} \mu \mathrm{m}^{-4}$. In method II, we directly calculate the density fluctuation (both configurational and vibrational ones are included) which causes the Rayleigh scattering of pure $\mathrm{SiO}_{2}$ glass. The density fluctuation is described by ${ }^{57,58}$,

$$
\left(\frac{\left\langle N^{2}\right\rangle-\left\langle N^{2}\right\rangle}{\langle N\rangle \bar{n}}\right)_{\mu \mathrm{VT}}=\left(\frac{\left\langle V^{2}\right\rangle-\left\langle V^{2}\right\rangle}{\langle V\rangle}\right)_{\mathrm{NPT}}=k_{\mathrm{B}} T \beta_{\mathrm{T}},
$$

where $N$ is the number of particles in volume $V, \bar{n}$ is the number density, $k_{B}$ is the Boltzmann's constant, $T$ is the temperature, $\beta_{\mathrm{T}}$ is the isothermal compressibility, and angle brackets indicate an average. $\mu \mathrm{VT}$ and NPT represent the grand canonical ensemble and the isothermal-isobaric ensemble, respectively. Because all MD simulations were carried out using the NPT ensemble, we have used volume variation of multiple glass samples instead of variation of the number of particles to calculate the density fluctuation. Data reported are from 18 samples prepared under similar conditions but different initial configurations.

\section{DATA AVAILABILITY}

Details of the simulations are available within the article. All data supporting the findings of this study are available from the authors upon reasonable request.

Received: 25 May 2020; Accepted: 26 August 2020; Published online: 17 September 2020 


\section{REFERENCES}

1. Varshneya, A. K. \& Mauro, J. C. Fundamentals of Inorganic Glasses, Ch. 1 (Elsevier, 2019).

2. Nishimura, Y. 'Electronic Industry History' 1981: Optical Fiber Communication, 156202 (Nikkei Electronics, 2008).

3. Miya, T., Terunuma, Y., Hosaka, T. \& Miyashita, T. Ultimate low-loss single-mode fiber at $1.55 \mu \mathrm{m}$. Electron. Lett. 15, 106-108 (1979).

4. Tamura, Y. et al. The first $0.14-\mathrm{dB} / \mathrm{km}$ loss optical fiber and its impact on submarine transmission. J. Light. Technol. 36, 44-49 (2018).

5. Tamura, Y. et al. Lowest-ever $0.1419-\mathrm{dB} / \mathrm{km}$ loss optical fiber. In Optical Fiber Communication Conference and Exhibition, 1-3 (2017).

6. Lee, H., Chen, T., Li, J., Painter, O. \& Vahala, K. J. Ultra-low-loss optical delay line on a silicon chip. Nat. Commun. 3, 1-7 (2012).

7. Takada, K., Yamada, H., Hida, Y., Ohmori, Y. \& Mitachi, S. Rayleigh backscattering measurement of $10 \mathrm{~m}$ long silica-based waveguides. Electron. Lett. 32, 1665-1667 (1996).

8. Miya, T. Silica-based planar lightwave circuits: passive and thermally active devices. IEEE J. Sel. Top. Quantum Electron. 6, 38-45 (2000).

9. Tsujikawa, K., Tajima, K. \& Ohashi, M. Rayleigh scattering reduction method for silica-based optical fiber. J. Light. Technol. 18, 1528-1532 (2000).

10. Olshansky, R. Propagation in glass optical waveguides. Rev. Mod. Phys. 51, 341-367 (1979)

11. Saito, K. et al. Limit of the Rayleigh scattering loss in silica fiber. Appl. Phys. Lett. 83, 5175-5177 (2003)

12. Saito, K., Ikushima, A. J., Ito, T. \& Itoh, A. A new method of developing ultralowloss glasses. J. Appl. Phys. 81, 7129-7134 (1997)

13. Anderson, J., Ellison, A. \& Schiefelbein, S. Method of doping silica glass with an alkali metal, and optical fiber precursor formed therefrom. U.S. Patent Application US2006/0130530 A1 (2006).

14. Kakiuchida, H., Saito, K. \& Ikushima, A. J. Rayleigh scattering in fluorine-doped silica glass. Jpn. J. Appl. Phys. 42, 6516-6517 (2003).

15. Ono, M., Aoyama, S., Fujinami, M. \& Ito, S. Significant suppression of Rayleigh scattering loss in silica glass formed by the compression of its melted phase. Opt. Express 26, 7942-7948 (2018).

16. Sato, T., Funamori, N., Wakabayashi, D., Nishida, K. \& Kikegawa, T. Coexistence of two states in optically homogeneous silica glass during the transformation in short-range order. Phys. Rev. B 98, 144111 (2018).

17. Guerette, $M$. et al. Structure and properties of silica glass densified in cold compression and hot compression. Sci. Rep. 5, 1-10 (2015).

18. Sundararaman, S., Huang, L., Ispas, S. \& Kob, W. New optimization scheme to obtain interaction potentials for oxide glasses. J. Chem. Phys. 148, 194504 (2018).

19. Meier, W. \& Moeck, H. The topology of three-dimensional 4-connected nets: classification of zeolite framework types using coordination sequences. J. Solid State Chem. 27, 349-355 (1979).

20. Brunner, G. O. The properties of coordination sequences and conclusions regarding the lowest possible density of zeolites. J. Solid State Chem. 29, 41-45 (1979).

21. Xue, X., Stebbins, J. F., Kanzaki, M., McMillan, P. F. \& Poe, B. Pressure-induced silicon coordination and tetrahedral structural changes in alkali oxide-silica melts up to 12 GPa: NMR, Raman, and infrared spectroscopy. Am. Mineral. 76, 8-26 (1991).

22. Zeidler, A., Salmon, P. S. \& Skinner, L. B. Packing and the structural transformations in liquid and amorphous oxides from ambient to extreme conditions. Proc. Natl Acad. Sci. U.S.A. 111, 10045-10048 (2014).

23. Koziatek, P., Barrat, J. L. \& Rodney, D. Short- and medium-range orders in as quenched and deformed $\mathrm{SiO}_{2}$ glasses: an atomistic study. J. Non-Cryst. Solids $\mathbf{4 1 4}$ 7-15 (2015).

24. Yuan, F. \& Huang, L. Brittle to ductile transition in densified silica glass. Sci. Rep. 4 5035 (2014).

25. Bohren, C. F. \& Huffman, D. R. Absorption and Scattering of Light by Small Particles, Ch. 5 (John Wiley \& Sons, 2008).

26. Pinnow, D. A., Rich, T. C., Ostermayer, F. W. \& DiDomenico, M. Fundamental optical attenuation limits in the liquid and glassy state with application to fiber optical waveguide materials. Appl. Phys. Lett. 22, 527-529 (1973).

27. Tan, C. Z., Arndt, J. \& Xie, H. S. Optical properties of densified silica glasses. Phys. $B$ Condens. Matter 252, 28-33 (1998).

28. Schroeder, J., Mohr, R., Macedo, P. B. \& Montrose, C. J. Rayleigh and Brillouin scattering in $\mathrm{K}_{2} \mathrm{O}-\mathrm{SiO}_{2}$ Glasses. J. Am. Ceram. Soc. 56, 510-514 (1973).

29. Stixrude, L. \& Bukowinski, M. S. T. A novel topological compression mechanism in a covalent liquid. Science 250, 541-543 (1990)

30. Stixrude, L. \& Bukowinski, M. Rings, topology, and the density of tectosilicates. Am. Mineral. 75, 1159-1169 (1990).

31. Saito, K., Kakiuchida, H. \& Ikushima, A. J. Investigation of the origin of the Rayleigh scattering in $\mathrm{SiO}_{2}$ glass. J. Non-Cryst. Solids 222, 329-334 (1997).
32. Brace, W. F., Schulz, C. H. \& La Mori, P. N. Isothermal compressibility of kyanite, andalusite, and sillimanite from synthetic aggregates. J. Geophys. Res. 74 2089-2098 (1969).

33. Hemley, R. J., Mao, H. K., Bell, P. M. \& Mysen, B. O. Raman spectroscopy of $\mathrm{SiO}_{2}$ glass at high pressure. Phys. Rev. Lett. 57, 747-750 (1986).

34. Trave, A., Tangney, P., Scandolo, S., Pasquarello, A. \& Car, R. Pressure-induced structural changes in liquid $\mathrm{SiO}_{2}$ from ab initio simulations. Phys. Rev. Lett. 89, 245504 (2002).

35. Karki, B. B., Bhattarai, D. \& Stixrude, L. First-principles simulations of liquid silica: Structural and dynamical behavior at high pressure. Phys. Rev. B 76, 104205 (2007).

36. Kubicki, J. D. \& Lasaga, A. C. Molecular dynamics simulations of $\mathrm{SiO}_{2}$ melt and glass: lonic and covalent models. Am. Mineral. 73, 941-955 (1988).

37. Galeener, F. L. Band limits and the vibrational spectra of tetrahedral glasses. Phys. Rev. B 19, 4292-4297 (1979).

38. Geissberger, A. E. \& Galeener, F. L. Raman studies of vitreous $\mathrm{SiO}_{2}$ versus fictive temperature. Phys. Rev. B 28, 3266-3271 (1983).

39. Deschamps, T. et al. Permanent densification of compressed silica glass: a Ramandensity calibration curve. J. Phys. Condens. Matter 25, 025402 (2013).

40. Bridgman, P. W. The compression of 39 substances to $100,000 \mathrm{~kg} / \mathrm{cm}^{2}$. Proc. Am. Acad. Arts Sci. 76, 55-70 (1948).

41. Bridgman, $P$. The compression of sixty-one solid substances to $25,000 \mathrm{~kg} / \mathrm{cm}^{2}$ determined by a new rapid method. Proc. Am. Acad. Arts Sci. 76, 9-24 (1945).

42. Bridgman, P. W. Certain physical properties of single crystals of tungsten, antimony, bismuth, tellurium, cadmium, zinc, and tin. Proc. Am. Acad. Arts Sci. 60, 305-383 (1925).

43. Vukcevich, M. R. A new interpretation of the anomalous properties of vitreous silica. J. Non-Cryst. Solids 11, 25-63 (1972).

44. Araki, S., Mohri, N., Yoshimitsu, Y. \& Miyake, Y. Synthesis, characterization and gas permeation properties of a silica membrane prepared by high-pressure chemical vapor deposition. J. Membr. Sci. 290, 138-145 (2007).

45. Singh, S., Ediger, M. D. \& de Pablo, J. J. Ultrastable glasses from in silico vapour deposition. Nat. Mater. 12, 139-144 (2013).

46. Nosé, S. A unified formulation of the constant temperature molecular dynamics methods. J. Chem. Phys. 81, 511-519 (1984)

47. Hoover, W. G. Canonical dynamics: equilibrium phase-space distributions. Phys. Rev. A 31, 1695-1697 (1985).

48. Hoover, W. G. Constant-pressure equations of motion. Phys. Rev. A 34, 2499-2500 (1986).

49. Carré, A., Berthier, L., Horbach, J., Ispas, S. \& Kob, W. Amorphous silica modeled with truncated and screened Coulomb interactions: a molecular dynamics simulation study. J. Chem. Phys. 127, 114512 (2007).

50. Van Beest, B., Kramer, G. J. \& Van Santen, R. Force fields for silicas and aluminophosphates based on ab initio calculations. Phys. Rev. Lett. 64, 1955-1958 (1990).

51. Plimpton, S. Fast parallel algorithms for short-range molecular dynamics. J. Comput. Phys. 117, 1-19 (1995).

52. Stukowski, A. Visualization and analysis of atomistic simulation data with OVITO-the open visualization tool. Model. Simul. Mater. Sci. Eng. 18, 015012 (2010).

53. Willems, T. F., Rycroft, C. H., Kazi, M., Meza, J. C. \& Haranczyk, M. Algorithms and tools for high-throughput geometry-based analysis of crystalline porous materials. Microporous Mesoporous Mater. 149, 134-141 (2012).

54. Pinheiro, M. et al. Characterization and comparison of pore landscapes in crys talline porous materials. J. Mol. Gr. Model. 44, 208-219 (2013).

55. Shannon, R. D. \& Prewitt, C. T. Effective ionic radii in oxides and fluorides. Acta Crystallogr. B 25, 925-946 (1969).

56. Le Roux, S. \& Jund, P. Ring statistics analysis of topological networks: new approach and application to amorphous $\mathrm{GeS}_{2}$ and $\mathrm{SiO}_{2}$ systems. Comput. Mater. Sci. 49, 70-83 (2010)

57. Watanabe, T., Saito, K. \& Ikushima, A. J. Fictive temperature dependence of density fluctuation in $\mathrm{SiO}_{2}$ glass. J. Appl. Phys. 94, 4824-4827 (2003).

58. Allen, M. P. \& Tildesley, D. J. Computer Simulation of Liquids, Ch. 2 (Oxford University Press, 2017)

\section{ACKNOWLEDGEMENTS}

Y.Y. and J.C.M. acknowledge financial support from AGC Inc. and the Institute for CyberScience Advanced CyberInfrastructure (ICS-ACI) at The Pennsylvania State University. Y.Y. acknowledges Siddharth Sundararaman for SHIK potential file and technical support from Christopher Blanton at ICS-ACI. We also thank Kazutaka Hayashi, Akio Koike, and Yuki Kondo from AGC Inc. for fruitful discussions. 


\section{AUTHOR CONTRIBUTIONS}

M.O. and J.C.M. designed the project. Y.Y. performed the molecular simulations, which Y.Y., M.O., S.U., and J.C.M. analyzed. O.H. performed the Raman spectroscopy experiments and analyzed the data. All authors participated in discussing the data. Y.Y., M.O., and J.C.M. wrote the paper with input from all authors.

\section{COMPETING INTERESTS}

The authors declare no competing interests.

\section{ADDITIONAL INFORMATION}

Supplementary information is available for this paper at https://doi.org/10.1038/ s41524-020-00408-1.

Correspondence and requests for materials should be addressed to M.O. or J.C.M.

Reprints and permission information is available at http://www.nature.com/ reprints
Publisher's note Springer Nature remains neutral with regard to jurisdictional claims in published maps and institutional affiliations.

Open Access This article is licensed under a Creative Commons adaptation, distribution and reproduction in any medium or format, as long as you give appropriate credit to the original author(s) and the source, provide a link to the Creative Commons license, and indicate if changes were made. The images or other third party material in this article are included in the article's Creative Commons license, unless indicated otherwise in a credit line to the material. If material is not included in the article's Creative Commons license and your intended use is not permitted by statutory regulation or exceeds the permitted use, you will need to obtain permission directly from the copyright holder. To view a copy of this license, visit http://creativecommons. org/licenses/by/4.0/.

(c) The Author(s) 2020 